\title{
Avaliação econômica dos casos de Dengue atribuídos ao desastre de 2011 em Nova Friburgo (RJ), Brasil
}

\author{
Economic evaluation of cases of dengue fever attributed to the \\ disaster of 2011 in Nova Friburgo (State of Rio de Janeiro/Brazil)
}

Carlos Alexandre Rodrigues Pereira ${ }^{1}$

Martha Macedo de Lima Barata ${ }^{1}$

Melania de Paulo Cariello Hoelz ${ }^{2}$

Viviane Nunes Lopes Oliveira Medeiros ${ }^{3}$

Felipe de Carvalho Vommaro Marincola ${ }^{1}$

Cristina Costa Neto ${ }^{1}$

Diana Pinheiro Marinho ${ }^{4}$

Teresa Vieira dos Santos Oliveira ${ }^{4}$

Aline Guimarães Monteiro Trigo ${ }^{5}$

Thiago Klein de Medeiros ${ }^{5}$

${ }^{1}$ Escola Nacional de Saúde Pública, Fiocruz. R. Leopoldo Bulhões 1480/812, Manguinhos. 21041-210 Rio de Janeiro RJ Brasil. carlos.rpereira@

hotmail.com

${ }^{2}$ Vigilância Epidemiológica, Fundação Municipal de Saúde de Nova Friburgo.

${ }^{3}$ Atenção Básica em Saúde, Fundação Municipal de Saúde de Nova Friburgo.

${ }^{4}$ Escola Nacional de Saúde Pública Sérgio Arouca,

Fiocruz.

${ }^{5}$ Departamento de Ensino, Centro Federal de Educação Tecnológica Celso Suckow da Fonseca.

\begin{abstract}
The prospects outlined in climate scenarios produced for Brazil indicate a probable increase in the number of extreme hydrometeorological events in the coming years. Therefore, a study of the health scourges that may intensify due to these events is important. The scope of this article is to estimate the cost represented by the cases of dengue fever attributed to the 2011 disaster in Nova Friburgo (RJ). There were 1,356 suspected cases of dengue fever, 937 of which were confirmed. The total cost of the disease may have been between $R \$ 66,000$ and $R \$ 499,000$ taking the minimum salary as a benchmark, with approximately $70 \%$ of this amount being among the confirmed cases. The disaster caused extensive changes in the city's environment which, together with the urban sanitation and clearing up process that occurred in the post-event period, led to an increase in the number of potential mosquito breeding sites, facilitating their proliferation and increasing the number of cases of dengue fever. This was a disease that in the decade prior to the disaster recorded few cases of the disease in the municipality. This illustrates the potential of events like the one that occurred in 2011 in Nova Friburgo may have on the increase in the number of cases, and consequently on the cost of the disease.
\end{abstract}

Key words Disaster evaluation, Cost of the disease, Health care costs
Resumo A perspectiva apontada pelos cenários climáticos desenvolvidos para o Brasil é de provável aumento no número de eventos hidrometeorológicos extremos nos próximos anos. Por isso, se faz importante o estudo dos agravos em saúde que poderão se intensificar em decorrência desses eventos. Portanto, por meio deste artigo, propõe-se estimar o custo representado pelos casos de dengue atribuídos ao desastre de 2011, em Nova Friburgo (RJ). Verificou-se que ocorreram 1.356 casos suspeitos de dengue, dos quais 937 foram confirmados. $O$ custo total da doença pode ter variado, no mínimo, entre R\$ 66 mil e R\$ 499 mil, no cenário de menor valor de salário, sendo que cerca de $70 \%$ desse custo ocorreu entre os casos confirmados. O desastre provocou grandes mudanças no ambiente do município que, junto com os problemas de saneamento e limpeza urbana, que ocorreram em períodos posteriores ao evento, acabou por aumentar a disponibilidade de locais que pudessem servir de criadouro do mosquito, facilitando sua proliferação e aumentando a incidência da dengue, doença que na década anterior ao desastre teve baixa incidência no município. Isso ilustra o potencial que eventos como ao ocorrido em 2011, em Nova Friburgo, podem ter no incremento desses casos $e$, consequentemente, nos custos da doença.

Palavras-chave Avaliação de desastres, Custos da doença, Custos de cuidados de saúde 


\section{Introdução}

Nova Friburgo é um dos municípios localizados na Região Serrana do Estado do Rio de Janeiro, apontada como uma das mais vulneráveis do estado a eventos extremos, como enchentes, inundações e deslizamentos'. Em Nova Friburgo, na noite do dia 11 para o dia 12 de janeiro de 2011, ocorreu forte chuva que durou cerca de cinco horas, com precipitação de $182,8 \mathrm{~mm}$. A chuva, associada a intensas descargas atmosféricas e fortes ventos, levou a escorregamentos de encostas em vários pontos, além da elevação do nível do Rio Bengalas, que corta a cidade. Devido a este evento, no município de Nova Friburgo 429 pessoas morreram e outras 3.220 ficaram desabrigadas. Diversos serviços foram afetados, como abastecimento de água, fornecimento de energia, transporte público e saúde². Dentre os impactos à saúde provocados pelo desastre, verificou-se o aumento no número de casos de dengue e leptospirose, doenças que foram alvo de medidas específicas de controle e notificação no município. A perspectiva apontada pelos cenários climáticos desenvolvidos para o Brasil é de provável aumento no número de eventos hidrometeorológicos extremos nos próximos anos, principalmente nas Regiões Sul e Sudeste ${ }^{3}$. Por isso, se faz importante o estudo dos agravos em saúde que poderão se intensificar em decorrência desses eventos. Portanto, por meio deste artigo propõe-se estimar o custo representado pelos casos de dengue atribuídos ao desastre de 2011 em Nova Friburgo (RJ), e discutir a relação da dengue com eventos dessa magnitude, apresentando parâmetros que possam ser utilizados no planejamento de ações de saúde e de adaptação e resposta aos desastres.

\section{Nova Friburgo}

O município é composto por oito distritos: Nova Friburgo, Riograndina, Campo do Coelho, Amparo, Lumiar, Conselheiro Paulino, São Pedro da Serra e Mury. Sua população é de aproximadamente 182 mil habitantes em uma área de pouco mais de $930 \mathrm{~km}^{2}$, correspondendo a uma densidade demográfica de $195 \mathrm{hab} / \mathrm{km}^{2}$. A maior parcela dos domicílios (29\%) pertence à classe C1, cuja renda familiar mensal é cerca de $\mathrm{R} \$ 1.400,00^{5}$. O Índice de Desenvolvimento Humano (IDH) do município é de $0,745^{4}$, enquanto que o IDH do Estado do Rio de Janeiro é de $0,761^{6}$. As principais atividades econômicas são: alimentação e bebidas (setor de serviços), artigos de vestuário e acessórios (comércio), confecção de roupas ínti- mas (setor industrial) e criação de bovinos (setor agropecuário) $)^{5}$. É um dos três municípios mais populosos da Região Serrana do Rio de Janeiro. No estudo realizado por Barata et al. ${ }^{1}$ sobre a vulnerabilidade da população dos municípios do Estado do Rio de Janeiro à mudança do clima, os municípios de Petrópolis, Teresópolis e Nova Friburgo se mostraram os mais vulneráveis a eventos hidrometeorológicos extremos, dentre os demais municípios da Região Serrana. Fatores naturais, como a própria paisagem e relevo, $\mathrm{e}$ fatores antrópicos, como a ocupação de áreas de encosta, cortes de taludes e retirada da vegetação nativa, podem contribuir para a sua vulnerabilidade a eventos hidrometeorológicos extremos.

\section{Dengue}

A dengue é uma doença infecciosa febril mundialmente difundida, cuja manifestação humana é provocada por um dos quatro sorotipos conhecidos do Arbovírus do gênero Flavivírus: DENV 1, 2, 3 ou $4^{7,8}$. Recentemente foi descoberto o sorotipo 5 , cuja infectividade para seres humanos ainda está sendo investigada9 O vetor da doença é o mosquito do gênero Aedes $^{8}$. Duas espécies são relatadas na transmissão da doença: Aedes aegypti (principal vetor nas Américas), e Aedes albopictus (relacionado a surtos da doença principalmente na Ásia), sendo as fêmeas do mosquito responsáveis pela transmissão, pois necessitam de sangue para maturar seus ovos. É uma doença febril aguda, que apresenta quatro tipos de manifestações clínicas: infecção inaparente, dengue clássico (DC), febre hemorrágica da dengue (FHD) ou síndrome do choque da dengue (SCD), sendo as duas últimas, as manifestações mais agravadas ${ }^{10}$. Acredita-se que a dengue seja uma doença cuja ocorrência possa ser facilitada por eventos extremos de chuva e pelas mudanças no clima $^{11}$. De acordo com Aström et al. ${ }^{12}$ o efeito do clima sobre a ocorrência da dengue ainda não é claro, mas sugere-se que a distribuição geográfica de seus vetores seja limitada pelas condições do clima, apesar de haver outros fatores que favorecem fortemente a ocorrência do agravo, como, segundo Rigau-Pérez et al. ${ }^{13}$, o crescimento demográfico descontrolado das cidades, o desconhecimento da população e a falta de estrutura dos serviços de saúde ${ }^{14}$. Além disso, os eventos com potencial destrutivo ao ambiente parecem criar condições propícias para a proliferação do vetor, como ocorreu durante a segunda guerra mundial, quando se ve- 


\section{Metodologia}

Este estudo é uma avaliação econômica parcial do tipo custo-doença relativa aos casos de dengue atribuídos ao desastre ocorrido em Nova Friburgo, na passagem do dia 11 para o dia 12 de janeiro de 2011. O município de Nova Friburgo foi escolhido para a pesquisa por ter sido um dos mais impactados pelo desastre de 2011, e por possuir registros das respostas em saúde adotadas, o que possibilitou a realização da avaliação econômica descrita. Dengue e leptospirose foram os agravos que mais se intensificaram após o desastre e que, no caso específico de Nova Friburgo, foram alvo de medidas específicas de controle epidemiológico e de notificação. Fez-se um levantamento dos casos de dengue que foram atribuídos ao desastre pelo serviço de saúde local. Avaliou-se os casos confirmados e os casos descartados. Optou-se por inserir os casos descartados na avaliação, pois, por mais que tenham sido rejeitados, por diagnóstico de outra doença ou por falta de informações, foram casos que tiveram a dengue como primeira suspeita, passando, portanto, pelo roteiro de investigação epidemiológica e gerando custos ao sistema.

\section{Fonte de Dados}

O estudo foi desenvolvido com base nos dados secundários de acesso restrito que foram cedidos pelos setores de Vigilância em Saúde e de Atenção Básica em Saúde da Fundação Municipal de Saúde de Nova Friburgo (FMS). Os dados de interesse foram retirados dos relatórios de vigilância epidemiológica, dos relatórios financeiros e de gestão e dos prontuários de notificação da dengue. A coleta de dados foi realizada entre dezembro de 2012 e abril de 2013.

\section{Custo-doença}

O custo de uma doença pode incluir tanto as despesas do sistema de saúde com o tratamento do agravo, quanto as despesas ou os prejuízos incorridos pela sociedade, como os dias de trabalho perdidos devido à doença ou os potenciais anos

de vida perdidos devido ao agravo. Dessa forma, agregando-se as parcelas do sistema de saúde e da sociedade, têm-se uma aproximação do custo real da doença, ou seja, do seu custo total. Esse custo total é chamado também de custo social. Mas vale lembrar que o custo social, neste sentido, não se refere apenas às parcelas da sociedade, mas sim ao custo total, inclusive do sistema de saúde.

\section{Custos ao Sistema de Saúde}

Os gastos públicos do setor de saúde no Brasil incluem aqueles efetuados pelo Sistema Único de Saúde (SUS), destinados ao equacionamento da doença. Neste componente de custo foram incluídas as despesas decorrentes de procedimentos hospitalares e ambulatoriais, que juntos representam os custos diretos médicos, e as despesas com acompanhante hospitalar, que representa o custo direto não médico. Os dados sobre custos diretos médicos foram retirados dos relatórios financeiros e de vigilância epidemiológica e dos prontuários de notificação da dengue. Já os custos com acompanhante foram calculados utilizando-se os valores constantes no Sistema de Gerenciamento da Tabela de Procedimentos, Medicamentos, Órteses, Próteses e Materiais Especiais do SUS (SIGTAP) ${ }^{15}$, para diárias de acompanhante. Foram estimados os custos de diárias de acompanhantes de pacientes cuja idade era menor de 18 anos ou maior ou igual a 60 anos, casos em que a permanência do acompanhante é permitida no âmbito do SUS ${ }^{16}$. Junto aos prontuários de notificação havia informações acerca dos exames diagnósticos realizados pelos pacientes. As quantidades e tipos de exames foram coletados nos prontuários, porém não foi possível estimar o custo de realização destes exames diagnósticos, pois, tanto no SIGTAP ${ }^{15}$ quanto no Sistema de Informações Ambulatoriais $(\mathrm{SIA})^{17}$, eles apareciam com custo igual à zero. Acredita-se que sejam disponibilizados aos laboratórios kits específicos para esses exames, o que não geraria custo adicional de realização. O laboratório que foi referência para Nova Friburgo também foi consultado, mas não se obteve informação sobre custos dos exames.

\section{Custos à Sociedade}

A sociedade também arca com parcelas do custo de uma doença, porém esses custos são chamados de indiretos. Neste estudo, os custos à sociedade foram representados pela perda de 
produtividade (PP), que é a força de trabalho que o paciente deixou de aplicar em suas atividades laborais devido ao absenteísmo pela doença. É um ônus ao empregador, uma vez que o empregado não deixa de receber pelos dias parados. Este custo pode ser calculado por meio da Equação 1, que é baseada em Motta ${ }^{18}$.

(Equação 1)

$P P=\left(\frac{\text { salário do trabalhador }+ \text { encargos sociais }}{\text { dias no mês }}\right) x$ dias parados

A perda de produtividade foi um dado estimado, uma vez que não há registros sobre absenteísmos ocorridos no município. No caso específico da dengue é recomendado que os pacientes permaneçam em repouso durante o tratamento, período em que a pessoa pode apresentar sintomas que dificultem a realização de atividades de vida diária ${ }^{19}$. Por isso, considera-se plausível esperar que tenham ocorrido absenteísmos entre os casos inseridos no estudo. Considerando-se que a PP seja o ônus ao empregador, que arca somente com os primeiros 15 dias de afastamento, poder-se-ia deixar de incluir os casos que por ventura tenham se afastado por períodos maiores que este. Contudo, tendo por base os aspectos clínicos da dengue clássica, manifestação mais frequente da dengue cuja duração, normalmente, varia de 5 a 7 dias ${ }^{10}$, poderia estar incluída no estudo a maior parte dos casos, restando apenas uma pequena minoria em que o período de convalescença tenha se prolongado por maior período devido à debilidade física do caso. Entre os casos hospitalares, a PP foi calculada com base no total de dias de internação. Entre os casos ambulatoriais, estimou-se uma faixa de valores cujo limite mínimo é o menor período possível de afastamento, ou seja, um dia, e cujo limite máximo considera a maior quantidade possível de dias de afastamento, ou seja, 15 dias. Para calcular a perda de produtividade total, utilizou-se dois cenários: um se refere ao período mínimo de absenteísmo conhecido, que é a perda de produtividade pelos dias de internação hospitalar. O outro se refere ao período máximo possível de absenteísmo, que ocorreria se todos os casos, ambulatoriais e hospitalares, tivessem se ausentado pelo maior período oneroso ao empregador, que é de 15 dias. Por fim, considerou-se como PP a faixa de valores compreendida entre os parâmetros calculados pelos dois cenários. Foram considerados como valores referência de salário: o salário mínimo nacional vigente em $2011^{20,21}$, o menor piso salarial do Estado do Rio de Janeiro em 201122; e o salário médio do município de Nova Friburgo ${ }^{23}$.

\section{Critérios Éticos}

Este artigo se refere a uma pesquisa aprovada pelo Comitê de Ética da Escola Nacional de Saúde Pública Sérgio Arouca - Ensp/Fiocruz e pelo Comitê de Ética do Instituto Oswaldo Cruz IOC/Fiocruz. Para a realização da pesquisa e coleta dos dados secundários foi assinado o Termo de Anuência pela FMS de Nova Friburgo e o Termo de Confidencialidade pelos pesquisadores.

\section{Resultados}

A dengue esteve fortemente relacionada ao desastre em função do número de casos, tanto confirmados quanto descartados, que ocorreram em Nova Friburgo naquele ano: foram 937 casos confirmados e 419 casos descartados da doença em 2011. Todos os casos ocorridos entre 20 de janeiro (data de início dos sintomas do primeiro caso verificado após o desastre) e 31 de dezembro de 2011 foram atribuídos ao desastre pela Vigilância em Saúde do município, porque os deslizamentos provocaram um desequilíbrio ambiental que favoreceu a manutenção e circulação dos vetores durante todo o ano. Acredita-se que os focos tenham surgido das casas abandonadas e dos escombros que ainda não haviam sido retirados. Esses criadouros naturais podem ter aumentado a densidade do mosquito e, com a aglomeração de pessoas em condições ambientais inadequadas, propiciado esse comportamento tão distinto da doença no município, inclusive com isolamento do vírus tipo 4 no Distrito de Riograndina durante o mês de fevereiro. Ao comparar o número de casos de 2011 com o de anos anteriores, desconsiderando anos de epidemia no Estado do Rio de Janeiro, se vê que o número de casos de 2011 foi bem maior e fora do perfil que normalmente ocorre no município, onde o pico começava em março, acentuava-se em abril e em maio caía, com poucos casos após esse período. Em 2011, apesar de ocorrerem esses picos, chegaram casos suspeitos de dengue aos serviços de saúde durante todo o ano. Ao todo, foram 1.356 casos suspeitos da doença, dos quais $69,1 \%$ (937 casos) foram confirmados. O Gráfico 1 ilustra a diferença no comportamento da doença nesse ano: nele pode ser verificada a quantidade de casos notificados no Sistema de Informação de Agravos de Notificação (SINAN) ${ }^{24}$, entre os anos de 2001 e 2011, onde se percebe o aumento do número de casos no ano do desastre. Analisando o local de ocorrência dos casos sus- 


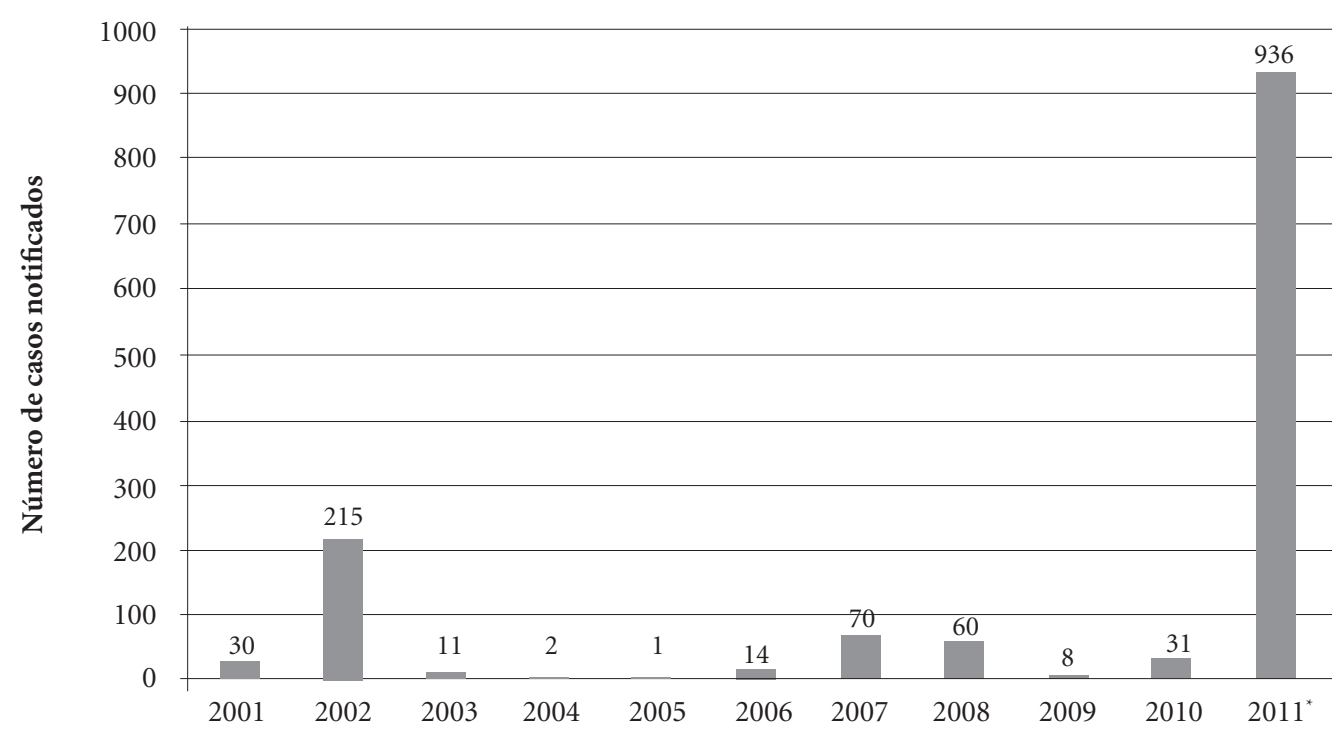

Gráfico 1. Casos notificados de dengue em Nova Friburgo entre 2001 e 2011.

“Dados de 2011 atualizados no sistema em 29/07/2013, sujeitos à revisão.

Fonte: SINAN ${ }^{23}$.

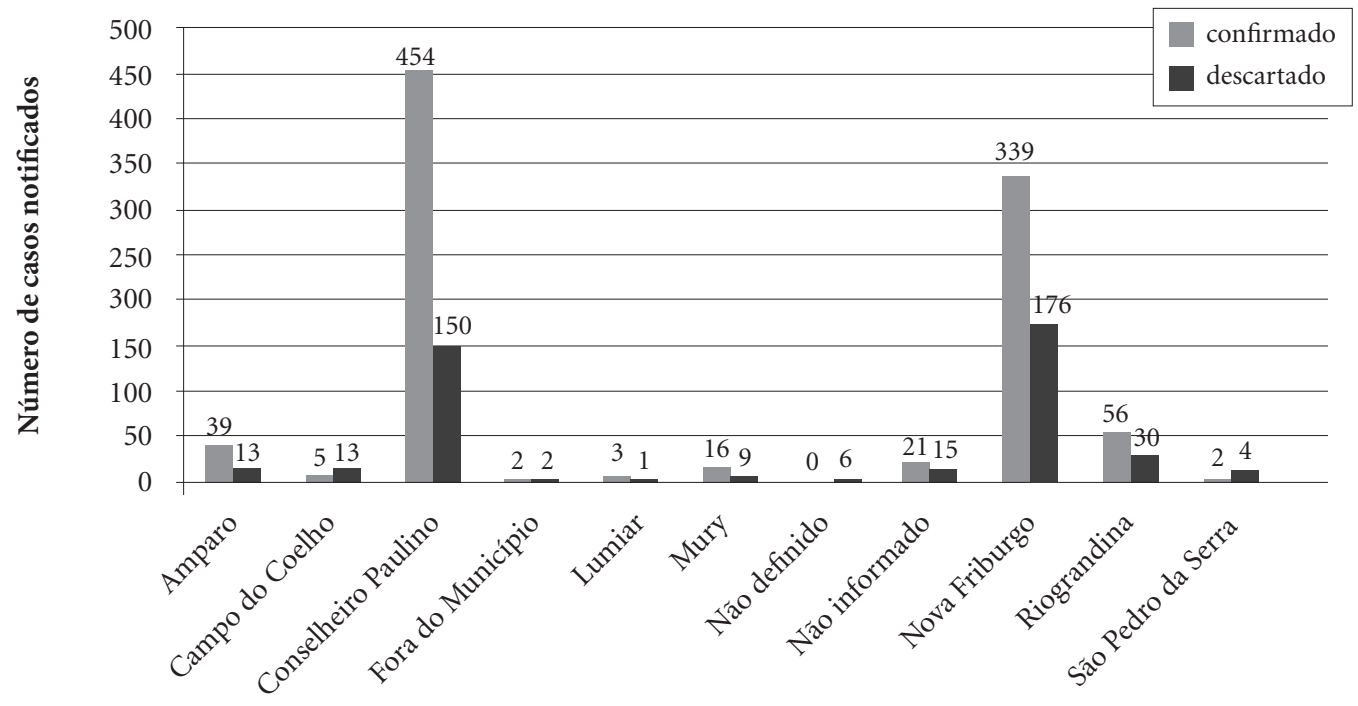

Gráfico 2. Número de casos suspeitos por classificação e distrito. 


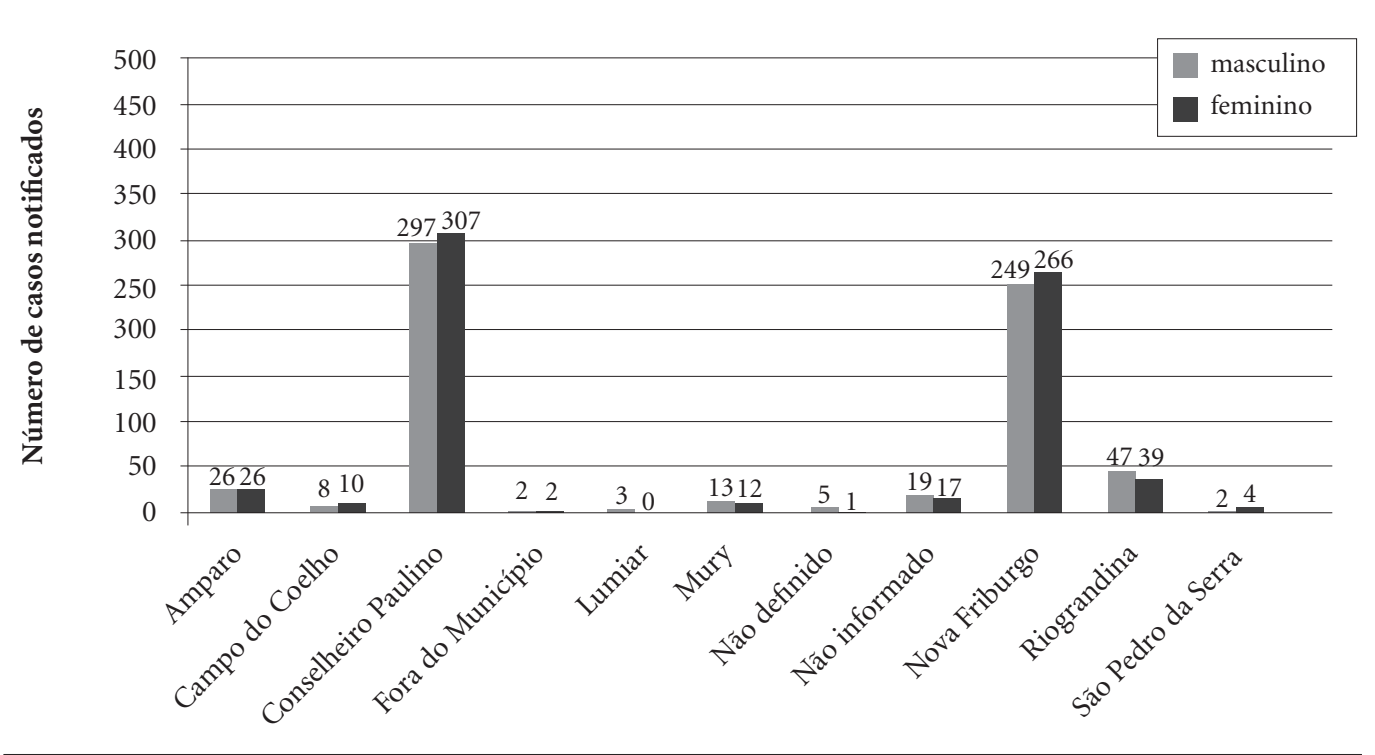

Gráfico 3. Número de casos suspeitos por sexo e distrito.

Fonte: Elaboração própria, com base nos dados coletados dos prontuários de notificação da doença.

Nos prontuários, todos os casos foram classificados como dengue clássico. Não foi verificado registro de complicação dos casos de dengue que tiveram confirmação diagnóstica. A respeito de exames laboratoriais, foram realizados ao todo 1.681 exames, sendo a pesquisa de anticorpos (IgM) o tipo de exame mais realizado. Esse exame é o recomendado para confirmação laboratorial na rotina ${ }^{10}$. Apesar de o isolamento viral ser o padrão ouro para o diagnóstico da dengue $^{10}$, ele foi realizado somente uma vez entre os casos estudados. A detecção de antígenos virais específicos de dengue do tipo NS1 também foi um exame muito aplicado, método que é recomendado em pesquisas e nos casos graves ${ }^{10}$. Outros exames como pesquisa de anticorpos IgG e reação em cadeia de polimerase transcriptase reversa (PCR) também foram realizados. Do total de 1.356 casos suspeitos, 101 receberam tratamento hospitalar e os demais 1.255 tiveram atendimento ambulatorial. Quanto aos casos que tiveram atendimento hospitalar, conforme pode ser visualizado na Tabela 1 , a idade mediana entre eles foi de 38 anos e a maioria foi classificada como sendo autóctone. Também em sua maioria, os casos eram do sexo feminino. Não foi verificado óbito por dengue entre estes pacientes. Dos 101 casos hospitalares, $78(77,23 \%)$ tiveram confirmação diagnóstica. Já entre os 1.255 casos suspeitos que receberam atendimento ambulatorial (Tabela 2), a idade mediana foi de 32 anos, e também a maioria era do sexo feminino. Aproximadamente 55\% desses casos foram classificados como autóctones, mas para aproximadamente $18 \%$ não foi possível determinar se a infecção ocorreu dentro ou fora do município. Também entre os casos ambulatoriais não foi verificado óbito por dengue, apesar de não haver informação sobre evolução para dois dos 1.255 casos inicialmente suspeitos. Do total de casos ambulatoriais suspeitos, 859 $(68,44 \%)$ tiveram confirmação diagnóstica.

\section{Custos ao Sistema de Saúde}

Foram gastos pelo Sistema de Saúde com o tratamento dos casos suspeitos, seja em ambiente ambulatorial ou hospitalar, o valor de $\mathrm{R} \$ 58.341,97$, sem considerar gastos com exames diagnósticos. Deste total, $\mathrm{R} \$ 45.791,97$ é referente ao gasto com casos suspeitos tratados em ambiente hospitalar, e R\$ 12.550,00 se refere àqueles tratados em ambulatório. Do montante utilizado para tratamento hospitalar da dengue, $\mathrm{R} \$ 34.622,58$ (75,61\%) foi o gasto incorrido entre os casos que receberam confirmação diagnóstica. Os R\$11.169,39 restantes $(24,39 \%)$ foram gastos com os casos hospitalares inicialmente suspeitos, mas que depois foram descartados. É válido considerar os casos 
Tabela 1. Estatísticas descritivas dos casos de dengue em Nova Friburgo que foram hospitalizados.

\begin{tabular}{|c|c|c|}
\hline Variável & $\mathrm{N}$ válido (\%) & Anos \\
\hline$n^{\circ}$ total de casos suspeitos & 101 & \\
\hline local de infecção & 101 & \\
\hline autóctone & $61(60,40)$ & \\
\hline fora do município & $37(36,63)$ & \\
\hline indeterminado & $3(02,97)$ & \\
\hline sexo & 101 & \\
\hline masculino & $48(47,52)$ & \\
\hline feminino & $53(52,48)$ & \\
\hline idade & 101 & \\
\hline média & & 40,90 \\
\hline mediana & & 38,00 \\
\hline desvio padrão & & 23,57 \\
\hline exames diagnósticos realizados & 174 & \\
\hline $\operatorname{IgM}$ & $67(38,51)$ & \\
\hline NS1 & $67(38,51)$ & \\
\hline isolamento viral & 0 & \\
\hline outros & $40(22,98)$ & \\
\hline evolução & 101 & \\
\hline cura & $98(97,03)$ & \\
\hline óbito por dengue & $0(0,00)$ & \\
\hline óbito por outras causas & $3(02,97)$ & \\
\hline $\mathrm{n}^{\circ}$ total de casos confirmados & 78 & \\
\hline $\begin{array}{l}\text { confirmados por critério } \\
\text { clínico/laboratorial }\end{array}$ & $71(91,03)$ & \\
\hline $\begin{array}{l}\text { confirmados por critério } \\
\text { clínico/epidemiológico }\end{array}$ & $7(08,97)$ & \\
\hline $\mathrm{n}^{\circ}$ total de casos descartados & 23 & \\
\hline $\begin{array}{l}\text { descartados por critério } \\
\text { clínico/laboratorial }\end{array}$ & $20(86,96)$ & \\
\hline $\begin{array}{l}\text { descartados por critério } \\
\text { clínico/epidemiológico }\end{array}$ & $3(13,04)$ & \\
\hline
\end{tabular}

descartados, pois a dengue foi sua primeira suspeita diagnóstica, o que levou a adoção do roteiro da investigação epidemiológica da doença, gerando, portanto, custos ao sistema de saúde. Do valor utilizado no tratamento ambulatorial dos casos suspeitos ( $\mathrm{R} \$ 12.550,00$ ), 68,45\% (R\$ $8.590,00)$ foi gasto entre os casos que tiveram confirmação diagnóstica. Os demais $31,55 \%$ (R\$ $3.960,00$ ) foram gastos entre os casos inicialmente suspeitos que foram descartados posteriormente.

\section{Custos à Sociedade}

Os custos à sociedade foram representados pela perda de produtividade, devido aos dias ausentes do trabalho por causa da doença. Como não há dados precisos sobre absenteísmos, foram dados estimados. Entre os casos hospitala-
Tabela 2. Estatísticas descritivas dos casos ambulatoriais de dengue em Nova Friburgo.

\begin{tabular}{|c|c|c|}
\hline Variável & N válido (\%) & Anos \\
\hline$n^{\circ}$ total de casos suspeitos & 1.255 & \\
\hline local de infecção & 1.253 & \\
\hline autóctone & $701(55,94)$ & \\
\hline fora do município & $317(25,30)$ & \\
\hline indeterminado & $235(18,76)$ & \\
\hline sexo & 1.255 & \\
\hline masculino & $624(49,72)$ & \\
\hline feminino & $631(50,28)$ & \\
\hline idade & 1.228 & \\
\hline média & & 34,49 \\
\hline mediana & & 32 \\
\hline desvio padrão & & 18,13 \\
\hline exames diagnósticos realizados & 1.507 & \\
\hline $\operatorname{IgM}$ & $542(35,96)$ & \\
\hline NS1 & $519(34,44)$ & \\
\hline isolamento viral & $1(00,07)$ & \\
\hline outros & $445(29,53)$ & \\
\hline evolução & 1.253 & \\
\hline cura & $1.252(99,92)$ & \\
\hline óbito por dengue & $0(0,00)$ & \\
\hline óbito por outras causas & $1(0,08)$ & \\
\hline $\begin{array}{l}\mathrm{n}^{\circ} \text { total de casos confirmados } \\
\text { confirmados por critério }\end{array}$ & 859 & \\
\hline $\begin{array}{l}\text { clínico/laboratorial } \\
\text { confirmados por critério }\end{array}$ & $623(72,53)$ & \\
\hline clínico/epidemiológico & $235(27,36)$ & \\
\hline $\mathrm{n}^{\circ}$ total de casos descartados & 396 & \\
\hline $\begin{array}{l}\text { descartados por critério } \\
\text { clínico/laboratorial }\end{array}$ & $272(68,69)$ & \\
\hline $\begin{array}{l}\text { descartados por critério } \\
\text { clínico/epidemiológico }\end{array}$ & $124(31,31)$ & \\
\hline
\end{tabular}

res confirmados, foram necessários 319 dias de internação, o que corresponde a uma média de 4,09 dias de internação por paciente. Considerando somente os 45 pacientes hospitalares que estavam em idade economicamente ativa, tem-se que estes demandaram 175 dias de internação, equivalente a 3,89 dias de internação, em média. Estima-se que estes casos economicamente ativos tiveram perda de produtividade (PP) de R\$5.652,60, quando é considerado o salário mínimo nacional de $2011^{20,21}$; $\mathrm{R} \$ 6.309,79$, quando se considera o salário mínimo estadual daquele ano; e $\mathrm{R} \$ 9.673,74$, com base na renda média de Nova Friburgo ${ }^{23}$.

Já entre os casos confirmados ambulatoriais, a perda de produtividade pode ter variado entre $\mathrm{R} \$ 20.820,00$ e $\mathrm{R} \$ 312.300,00$, faixa de valores cujos limites representam a PP que ocorreria 
se todos os casos se ausentassem por um único dia e a PP que ocorreria se todos os casos se ausentassem pelo período máximo de 15 dias, respectivamente. Esse valor foi calculado utilizando-se o salário mínimo nacional de $2011^{20,21}$. Quando se utiliza o salário mínimo estadual daquele ano ${ }^{22}$ nos cálculos, essa PP passa a variar entre $\mathrm{R} \$ 23.233$, 17 e $\mathrm{R} \$ 348.497,60$; Já com base na renda média de Nova Friburgo ${ }^{23}$, a PP passa a variar entre $R \$ 35.619,51$ e $R$ \$ 534.292,67. Agregando-se as estimativas de perda de renda ocorrida somente entre os casos confirmados de dengue, tanto hospitalares quanto ambulatoriais, tem-se que esta pode ter variado de $\mathrm{R} \$ 5.652,60 \mathrm{e}$ $\mathrm{R} \$ 313.764,75$, considerando-se o salário mínimo nacional de $2011^{19,20} ; \mathrm{R} \$ 6.309,79$ e $\mathrm{R} \$ 350.138,88$, quando se considera o salário mínimo estadual daquele ano ${ }^{21}$; e $\mathrm{R} \$ 9.673,74$ e $\mathrm{R} \$ 536.808,96$, com base na renda média de Nova Friburgo ${ }^{23}$. Com relação à PP ocorrida entre os casos descartados, tem-se que, entre aqueles em idade economicamente ativa tratados em ambiente hospitalar (oito casos), ocorreram 89 dias de internação, correspondendo a 11, 12 dias por paciente. Para estes casos, a PP estimada foi de R $\$ 2.413,50$, no cenário de menor valor de salário (salário mínimo nacional); R\$2.698,99, no cenário de valor de salário intermediário (salário mínimo estadual); e R \$ 4.137,90, no cenário de maior valor de salário (renda média do município). A PP total para os casos descartados, tanto hospitalares quanto ambulatoriais, variou de $\mathrm{R} \$ 2.413,50$ a $\mathrm{R} \$$ 127.187,40, no cenário de menor valor de salário; $\mathrm{R} \$ 2.698,99$ a $\mathrm{R} \$ 141.988,61$, no cenário intermediário de valor de salário; e de $\mathrm{R} \$ 4.137,90$ a $\mathrm{R} \$$ 217.687,22, no cenário de maior valor de renda. A PP geral, somando-se aquela ocorrida entre casos confirmados e aquela ocorrida entre os casos descartados da doença, sejam eles ambulatoriais ou hospitalares, pode ter variado entre $\mathrm{R} \$ 8.066,10$ e $\mathrm{R} \$ 440.952,15$, no cenário de menor valor de salário; $\mathrm{R} \$ 9.008,78$ e $\mathrm{R} \$ 492.127,49$, no cenário intermediário de valor de salário; $\mathrm{R} \$$ 13.811,64 e R\$ 754.496,18, no cenário de maior valor de renda.

\section{Custo Total da Doença}

Somando-se os custos atribuídos ao sistema de saúde e os custos da sociedade, verifica-se que somente os casos confirmados de dengue podem ter representado um custo que variou de $\mathrm{R} \$$ $48.865,18$ a $\mathrm{R} \$ 356.977,33$, no cenário de menor valor de salário; $\mathrm{R} \$ 49.522,37$ a $\mathrm{R} \$ 393.351,46$, no cenário de valor intermediário de salário; e R\$
$52.886,32$ a R \$ 580.021,54, no cenário de maior valor de renda. Este custo ocorrido entre os casos confirmados de dengue, tanto ambulatoriais quanto hospitalares, representa cerca de $70 \%$ do custo total da doença, que variou de $\mathrm{R} \$ 66.408,07$ a R $\$ 499.294,12$, no cenário de menor valor de salário; $\mathrm{R} \$ 67.350,75$ a R \$ 550.469,46, no cenário de valor intermediário de salário; e R \$ 72.153,61 a $R \$ 812.838,15$, no cenário de maior valor de renda. Verificou-se que a maior parcela do custo total da doença foi absorvida pela sociedade.

\section{Discussão}

O Banco Mundial ${ }^{25}$ estimou as perdas e danos provocados pelo desastre de 2011 na Região Serrana do Estado do Rio de Janeiro em cerca de $\mathrm{R} \$ 4,78$ bilhões, dos quais aproximadamente $\mathrm{R} \$$ 3,15 bilhões foram atribuídos ao setor público e $\mathrm{R} \$ 1,62$ bilhões ao setor privado. Os valores se referem à soma das perdas e danos de todos os municípios da região que foram afetados. Não foram apresentadas estimativas por município, não sendo possível, portanto, visualizar os custos incorridos somente em Nova Friburgo. Nesse mesmo estudo ${ }^{25}$, foram estimadas perdas e danos ao setor de saúde na ordem de $\mathrm{R} \$ 11$, 27 milhões, mas a falta de informações impossibilitou uma avaliação mais precisa do custo a este setor. Não foram incluídos nas estimativas os custos com as doenças relacionadas ao desastre, nem os custos de atividades de vigilância e de atenção básica.

Eventos extremos em Nova Friburgo são recorrentes, conforme relataram Freitas et al. ${ }^{26}:$ em 1988, enchentes atingiram Nova Friburgo, Petrópolis e Teresópolis e provocaram 227 óbitos e 2 mil desabrigados. Em 2000, enchentes em Nova Friburgo, Petrópolis e Teresópolis ocasionaram cinco óbitos. Em 2007, chuvas fortes em Nova Friburgo, Sumidouro, Petrópolis e Teresópolis resultaram em 23 óbitos, dos quais, segundo Barata et al. ${ }^{1}$ ocorreram em Nova Friburgo. Barata et al. ${ }^{1}$ ainda relataram que inundações bruscas em 2005 provocaram um óbito no município.

Freitas et al. ${ }^{26}$ ainda destacam a vulnerabilidade socioambiental e dos serviços de saúde nos municípios da Região Serrana, local onde problemas crônicos de infraestrutura e de planejamento urbano aumentam o potencial para perdas e danos ambientais, sociais e de saúde. Em decorrência do desastre, houve em Nova Friburgo mais casos confirmados de dengue que de leptospirose, que também foi associada ao desastre no município: foram 937 casos 
confirmados de dengue e 177 de leptospirose ${ }^{27}$. Tomando como referência somente os casos confirmados de dengue e de leptospirose ${ }^{27}$ ocorridos no município e associados ao desastre, tem-se que: o custo social da dengue pode ter chegado a $\mathrm{R} \$ 580$ mil e da leptospirose a $\mathrm{R} \$ 159$ mil; a proporção ambulatorial/hospitalar foi de 11,01 casos ambulatoriais para cada caso hospitalar de dengue e de 5,32 casos ambulatoriais para cada caso hospitalar de leptospirose; o tempo médio de internação foi de 4,13 dias para dengue e de 6,36 dias para leptospirose; o custo médio hospitalar para os casos de dengue foi de $\mathrm{R} \$ 443,88$ e de leptospirose foi de $\mathrm{R} \$ 603,63$. É relevante mencionar estes resultados porque relatos de casos de dengue atribuídos a desastres são menos comuns que relatos de casos de leptospirose, que é mais frequentemente associada a desastres e a eventos extremos de chuva. Os resultados deste estudo podem sinalizar a necessidade de aprofundar a abordagem sobre os efeitos de eventos extremos e desastres ambientais para o incremento de casos de dengue e o estudo do custo social desta doença, que se mostrou relevante no contexto de Nova Friburgo.

Fanany ${ }^{28}$, em seu estudo sobre os casos de febre hemorrágica da dengue (FHD) ocorridos em Padang, West Sumatra, Indonésia, após terremoto ocorrido em setembro de 2009, relatou que o desastre provocou o aumento no número de casos desta manifestação clínica da dengue, interferindo no comportamento da doença também em períodos posteriores. Relata ainda que, historicamente na Indonésia, casos de dengue se relacionam fortemente com precipitação, mas a temperatura aparece como um dos fatores que levam a severidade dos surtos, além do que, fatores locais como vegetação, tipo de construção, densidade populacional e disposição de lixo, influenciam na ocorrência da doença, transmitida principalmente pelos vetores Aedes aegypti e Aedes albopictus. Segundo o autor, por mais que a dengue seja um problema crônico da área, o terremoto de setembro de 2009 criou um cenário que facilitou a transmissão da doença e que pode ter dificultado as ações de prevenção. O grande número de lotes abandonados e a dificuldade de limpeza dos escombros e os acúmulos irregulares de água alteraram o ambiente local de tal forma que propiciou a reprodução dos mosquitos.

Em Nova Friburgo não foi verificado caso de FHD que tivesse sido atribuído ao desastre de 2011, mas foi nítido o aumento no número de casos de Dengue Clássico. Pelo que se pode perceber na leitura do trabalho de Fanany ${ }^{28}$, o cenário pós-desastre instalado em Padang, no que se refere a circunstâncias facilitadoras da transmissão da dengue, foi relativamente próximo ao que se verificou em Nova Friburgo. Ambos os casos evidenciaram o efeito que eventos que levam a desmoronamentos, movimentos de massa e queda de edifícios podem ter sobre a epidemiologia da dengue. Sendo a dengue uma doença sensível às condições sanitárias, principalmente de limpeza urbana 10 - o que dificilmente é possível manter em níveis ideais imediatamente após um evento de crise como um desastre -, a permanência de áreas de escombros pode ter contribuído para o aumento no número de casos de dengue no período pós-desastre em Nova Friburgo, comparado com períodos anteriores.

Miranda et al. ${ }^{29}$ relatam que no Brasil, a doença possui um padrão no qual a cada dois ou três anos ocorre aumento no número de casos com posterior redução acentuada, sendo 2002 um ano característico de aumento de casos em todo o Brasil. Os autores também relatam que em 2007 houve muitos casos, principalmente na região Sudeste do Brasil, e que em 2008 ocorreu no Estado do Rio de Janeiro mais de 150 mil casos somente entre os meses de janeiro a maio. Conforme mostrado no Gráfico 1, os anos de 2000, 2007 e 2008 também foram anos de maior notificação de casos em Nova Friburgo, o que é condizente com os períodos de surto apresentados por estes autores ${ }^{29}$. Seguindo esse padrão de comportamento da doença, não seria esperado, por natureza do agravo, um surto em 2011, o que reforça ainda mais o impacto do desastre para o incremento dos casos de dengue no município.

Miranda et al. ${ }^{29}$ ainda atentam para o fato de que o acesso aos serviços de saúde e a qualidade dos mesmos podem interferir no impacto de uma epidemia. Com relação ao acesso e à qualidade dos serviços de saúde, muitas unidades públicas de saúde foram atingidas pelo desastre, havendo necessidade de montar hospital de campanha e de adaptar a maternidade que funcionou como hospital geral ${ }^{2}$.

A estimativa de custo social alcançada pode servir de parâmetro para o planejamento de ações de saúde e de adaptação e resposta a desastres. O uso de informação apoia na tomada de decisão e contribui para a eficácia da gestão pública $^{30}$. Conhecendo-se o impacto financeiro de uma doença é mais fácil avaliar o custo-benefício de projetos e programas voltados à sua redução. E neste caso, em que o custo social da doença é parte do custo social do desastre, poder-se-ia 
também utilizar esse parâmetro na análise de estratégias voltadas à adaptação e resposta a esses eventos, uma vez que os sistemas públicos devem se responsabilizar pela implementação de políticas de prevenção e minimização dos efeitos de desastres, não só sobre a saúde.

Segundo Fensterseifer ${ }^{31}$, o Estado deve se responsabilizar por danos causados por eventos extremos e buscar meios de indenizar aos atingidos e atender aos seus direitos básicos, principalmente quando a situação de vulnerabilidade resulta da própria omissão estatal em prevenir danos resultantes das mudanças climáticas. Os resultados apresentados encontram limitação na quantidade de variáveis inseridas no modelo: quanto mais variáveis, maior o custo estimado e mais próximo se estaria do custo real. Porém, o uso de muitas variáveis deixaria o modelo complexo a ponto de dificultar as análises. Por isso, optou-se por utilizar as variáveis de maior interesse para a sociedade e para o sistema público de saúde. É relevante considerar para essa pesquisa o princípio da indeterminação (incerteza): não se alcançará a verdade definitiva, mas uma estimativa aceitável para que determinados processos possam ser compreensíveis. Também o conteúdo das fontes de dados é sensível aos problemas de diagnóstico de dengue. As próprias condições de notificação, na situação de desastre, podem favorecer a subnotificação de casos. Sendo assim, casos subnotificados podem levar a uma subestimativa de custos. Contudo, acredita-se que, ainda assim, a obtenção de uma estimativa do custo social das doenças atribuídas ao desastre ocorrido em Nova Friburgo, em 2011, possa ser dado útil para interação entre as partes interessadas (academia, gestores e comunidade), e para melhoria nas práticas de gestão em saúde e prevenção de desastres.

\section{Considerações finais}

Relatou-se neste trabalho a estimativa do custo social dos casos de dengue atribuídos ao desastre de janeiro de 2011 em Nova Friburgo (RJ/Brasil). Verificou-se que ocorreram 1.356 casos suspeitos de dengue, dos quais 937 foram confirmados. $\mathrm{O}$ custo total da doença pode ter variado aproxima- damente entre $\mathrm{R} \$ 66.000,00$ e $\mathrm{R} \$ 499.000,00$, no cenário de menor valor de salário; $\mathrm{R} \$ 67.000,00$ e $\mathrm{R} \$ 550.000,00$, no cenário de valor intermediário de salário; R $\$ 72.000,00$ e R\$813.000,00, no cenário de maior valor de renda, sendo que cerca de $70 \%$ desse custo ocorreu entre os casos confirmados de dengue, tanto ambulatoriais quanto hospitalares. Observou-se, ainda, que a maior parcela do custo total da doença foi absorvida pela sociedade através da perda de produtividade. O desastre provocou grandes mudanças no ambiente do município que, junto com os problemas de saneamento e limpeza urbana, ocorridos em períodos posteriores ao evento, aumentou a disponibilidade de locais que pudessem servir de criadouro do mosquito, facilitando sua proliferação e aumentando a incidência da dengue após o desastre. Isso ilustra o potencial que eventos como o ocorrido em 2011 em Nova Friburgo podem ter em incrementar os casos e, consequentemente, os custos da doença.

A medida da carga financeira e social de uma doença é uma informação que pode ser útil, para que a gestão em saúde e ambiente possa decidir onde e como aplicar seus recursos, e que justifica a necessidade de investimento e fortalecimento do setor de Vîgilância em Saúde, que é fundamental para o controle de surtos e miminização dos efeitos de dasastres sobre a saúde. Além disso, as avaliações de custo social de desastres podem subsidiar o Estado na alocação de medidas prestacionais à população afetada.

Com base nos resultados alcançados, acredita-se ser necessário aprofundar a pesquisa sobre o efeito de desastres sobre a epidemiologia da dengue, doença que, mesmo sendo associada a desastres, nem sempre é alvo de avaliações epidemiológicas ou econômicas, que poderiam levar a um melhor entendimento da relação entre desastres, ou às modificações ambientais trazidas por eles, e a ocorrência da doença. Espera-se que este trabalho possa incentivar a pesquisa sobre o custo social de desastres e na avaliação de doenças que, como a dengue, podem ocorrer após estes eventos, seja a curto, médio ou longo prazos. É preciso avançar na perspectiva de estudos práticos que possam ser aplicados no cotidiano da gestão pública em saúde ambiental. 


\section{Colaboradores}

CAR Pereira, MML Barata, MPC Hoelz, VNLO Medeiros, FCV Marincola, C Costa Neto, DP Marinho, TVS Oliveira, AGM Trigo e TK Medeiros participaram igualmente de todas as etapas de elaboração do artigo.

\section{Referências}

1. Barata MML, Confalonieri UEC, Lima ACL, Marinho DP, Luigi G, De Simone GC, Ferreira IB, Pinto IV, Tosta FO, Silva HVO, Valadares AS. Mapa de vulnerabilidade da população do estado do Rio de Janeiro aos impactos das mudanças climáticas nas áreas social, saúde e ambiente: Relatório Executivo $n^{\circ} 4$. Rio de Janeiro: Fundação Oswaldo Cruz, Secretaria de Estado do Ambiente do Rio de Janeiro; 2011.

2. Porto SS. Saúde em desastres: a experiência de Nova Friburgo [CD-ROM]. 2012.

3. Marengo JA, Nobre CA, Salati E, Ambrizzi T. Caracterização do clima atual e definição das alterações climáticas para o território brasileiro ao longo do Século XXI. Brasília: Ministério do Meio Ambiente; 2007.

4. Brasil. Instituto Brasileiro de Geografia e Estatística. Cidades@ [base de dados]. [Online]; 2012 [acessado 2013 out 02]. Disponível em: http://cidades.ibge.gov. br/xtras/home.php

5. Serviço Brasileiro de Apoio às Micro e Pequenas Empresas (Sebrae/RJ). Informações socioeconômicas do município de Nova Friburgo. Rio de Janeiro: Sebrae; 2011.

6. Instituto Brasileiro de Geografia e Estatística (IBGE). Estados@ [base de dados]. [Online]; 2012 [acessado 2013 out 02]. Disponível em: http://www.ibge.gov.br/ estadosat/temas.php?sigla $=$ rj\&tema $=i d h m$

7. Calisher CH, Karabatsos N, Dalrymple JM, Shope RE, Porterfield JS, Westaway EG, Brandt WE. Antigenic Relationships between Flaviviruses as Determined by Cross-neutralization Tests with Polyclonal Antisera. J Gen Virol 1989; 70(Pt.1):37-43.

8. Racloz V, Ramsey R, Tong S, Hu W. Surveillance of Dengue Fever Virus: A Review of Epidemiological Models and Early Warning Systems. PLoS Negl Trop Dis 2012; 6(5):e1648.

9. Sociedade Brasileira de Medicina Tropical. Dengue tipo 5 é descoberta em país tropical. [Online]; 2013 [acessado 2014 jan 10]. Disponível em: http://sbmt.org.br/site/ corpo_texto/2557\#.UvzEDPldWIA

10. Brasil. Guia de Vigilância Epidemiológica. $7^{\text {a }}$ ed. Brasília: MS; 2009.

11. Jack S. Injusticia cósmica: el brote de dengue en América Latina es un presagio ominoso. Latin Trade 2002; 22(jun).

12. Aström C, Rocklöv J, Hales S, Béguin A, Louis V, Sauerbor R. Potential Distribution of Dengue Fever Under Scenarios of Climate Change and Economic Development. EcoHealth 2012; 9(4):448-454.

13. Rigau-Pérez JG, Clark GG, Gubler DJ, Reiter P, Sanders EJ, Vorndam AV. Dengue and dengue haemorrhagic fever. Lancet 1998; 352(9132):971-977.

14. Reis CB, Andrade SMO, Cunha RV. Aliados do A. Aegypti: fatores contribuintes para a ocorrência do dengue segundo as representações sociais dos profissionais das equipes de saúde da família. Cien Saude Colet 2013; 18(2):517-526.

15. Brasil. Sistema de Gerenciamento da Tabela de Procedimentos, Medicamentos, Órteses, Próteses e Materiais Especiais do SUS [base de dados]. [Online]. [acessado 2013 ago 9]. Disponível em: http://sigtap.datasus.gov. br/tabela-unificada/app/sec/inicio.jsp . 
16. Instituto Brasileiro de Defesa do Consumidor (IDEC). Guia do direito à saúde: Sistema Público de Saúde (SUS), medicamentos e planos de saúde. [Online]. [S.l.: sn]; 2006 [acessado 2012 out 12]. Disponível em: http://www. guiadedireitos.org/downloads/guia_direito_saude

17. Brasil. Sistema de Informações Ambulatoriais [base de dados]. [Online]. [acessado 2013 set 13]. Disponível em: http://w3.datasus.gov.br/siasih/siasih.php.

18. Motta R. Manual para valoração econômica de recursos ambientais. Rio de Janeiro: IPEA; 1997.

19. Universidade de São Paulo, Departamento de Química. Portal da Dengue. [Online]. [acessado 2012 out 30]. Disponível em: http://portaldadengue.ffclrp.usp. br/?page_id $=517$.

20. Brasil. Medida Provisória no 516 de 30 de dezembro de 2010. Dispõe sobre o valor do salário mínimo nacional de 2011. Diário Oficial da União 2010; $31 \mathrm{dez}$.

21. Brasil. Lei no 12.382 de 25 de fevereiro de 2011. Dispõe sobre o valor do salário mínimo nacional de 2011. Diário Oficial da União 2011; 28 fev.

22. Estado do Rio de Janeiro. Lei no 5.950 de 13 de abril de 2011. Institui pisos salariais, no âmbito do estado do rio de janeiro para as categorias profissionais que menciona e estabelece outras providências. Diário Oficial do Executivo 2011; 14 abr.

23. Fundação Getúlio Vargas, Centro de Políticas Sociais. Renda Média. [Online]. [2011?] [acessado 2013 out 15]. Disponível em: http://www.cps.fgv.br/cps/bd/nbrics/ NBRICS_Rankings_Listagem-ultimorenda_site.xlsx .

24. Brasil. Sistema de Informação de Agravos de Notificação [base de dados]. [Online]. [acessado $2013 \mathrm{dez} 12$ ]. Disponível em: http://dtr2004.saude.gov.br/sinanweb/

25. Banco Mundial (BM). Avaliação de perdas e danos: inundações e deslizamentos na Região Serrana do Rio de Janeiro - Janeiro de 2011. Brasília: BM; 2012.
26. Freitas CM, Carvalho ML, Ximenes EF, Arraes EF, Gomes JO. Vulnerabilidade socioambiental, redução de riscos de desastres e construção da resiliência - lições do terremoto no Haiti e das chuvas fortes na Região Serrana, Brasil. Cien Saude Colet 2012; 17(6):15771586.

27. Pereira CAR, Barata MML, Trigo AGM. Social Cost of Leptospirosis Cases Attributed to the 2011 Disaster Striking Nova Friburgo, Brazil. Int J Environ Res Public Health 2014: 11(4):4140-4157.

28. Fanany D. Dengue Hemorrhagic Fever and Natural Disaster: The Case of Padang, West Sumatra. IJCRIMPH 2012; 4(5):673-678.

29. Miranda ES, Osório-de-Castro CGS, Santos-Pinto CDB. Preparo e resistência desastres no Brasil: experiências da dengue e da malária. Saúde debate 2011; 35(88):138-147.

30. Barata MML, Kligerman DC, Minayo C. A Gestão Ambiental no Setor Público: Uma Questão de Relevância Social. Cien Saude Colet 2006; 12(1):1-10.

31. Fensterseifer T. A responsabilidade do estado pelos danos causados às pessoas atingidas pelos desastres ambientais ocasionados pelas mudanças climáticas: uma análise à luz dos deveres de proteção ambiental do estado e da correspondente proibição de insuficiência na tutela do direito fundamental ao ambiente. [Online]; 2011 [acessado 2013 dez 10]. Disponível em: http://www.defensoria. sp.gov.br/dpesp/Repositorio/31/Documentos/12.pdf

Artigo apresentado em 14/03/2014

Aprovado em 01/06/2014

Versão final apresentada em 02/06/2014 